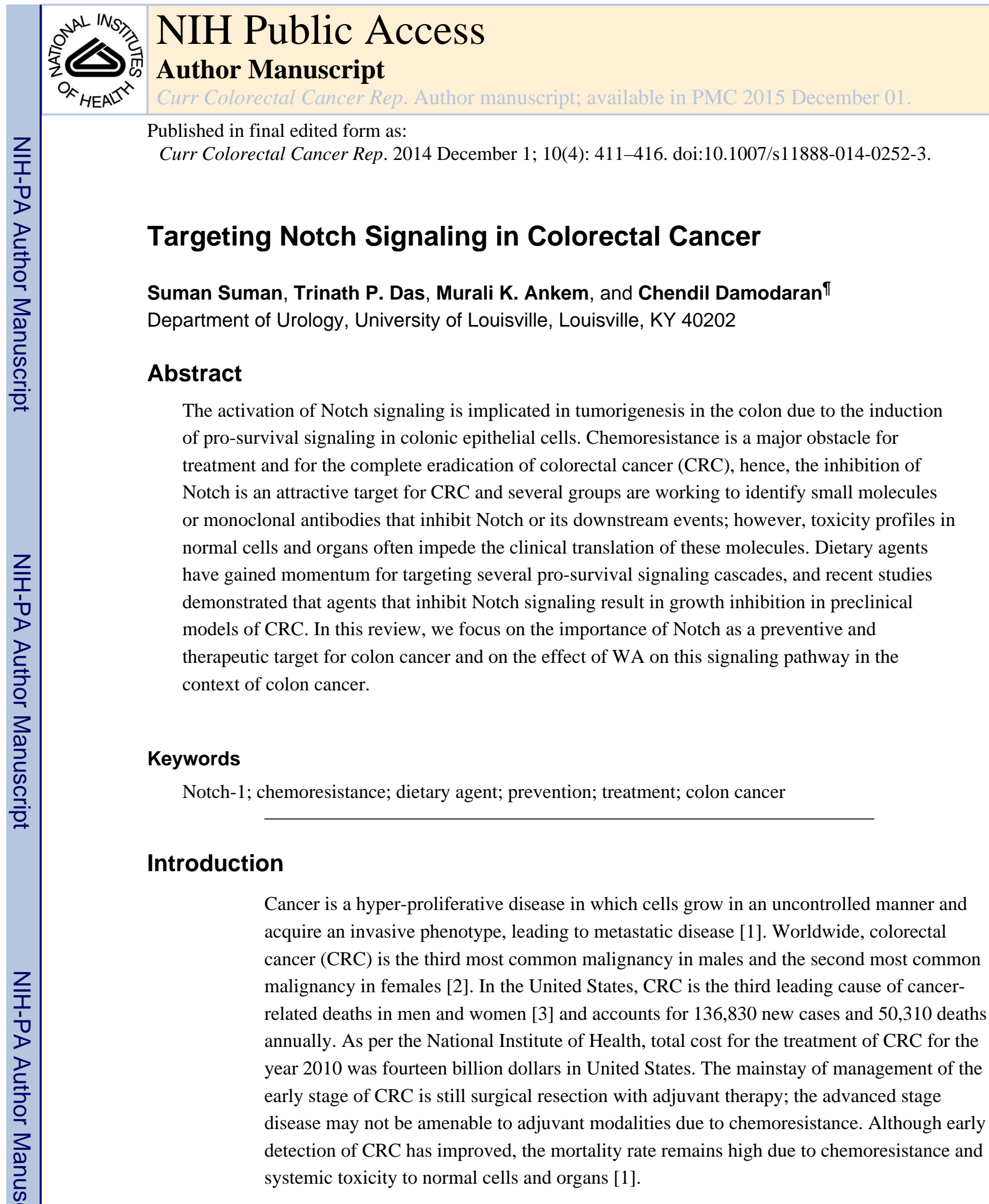

ICorrespondence to: Chendil Damodaran, Department of Urology, University of Louisville, Louisville, KY 40202, Tel: 502-852-5229, Fax: 502-852-2123, chendil.damodaran@louisville.edu.

Conflict of Interest

Suman Suman, Trinath P. Das, Murali. K. Ankem, and Chendil Damodaran declare that they have no conflict of interest.

Compliance with Ethics Guidelines

Human and Animal Rights and Informed Consent

This article does not contain any studies with human or animal subjects performed by any of the authors. 
CRC is a multistep process that involves the disruption of molecular mechanisms necessary for intestinal homeostasis by maintaining intestinal proliferation, differentiation and programmed cell death. The repercussions of this deregulation of cellular signals promotes oncogenic phenotypes, in which cells exhibit uncontrolled proliferation, the loss of apoptosis, a highly invasive phenotype that advances to metastasis, the induction of angiogenesis and chemoresistance to drugs [4]. It is imperative to understand the molecular mechanisms involved in the development and progression of CRC to identify novel targets and develop novel drugs, which could benefit patients with CRC by avoiding the disadvantages associated with current treatment modalities. This review article focuses on the potential of the natural compound Withaferin A (WA) and its ability to target Notch signaling and impede CRC development and progression.

\section{Notch signaling and components}

The Notch signaling pathway consists of five Notch ligands (i.e., the Delta-like ligands DLL1, DLL3 and DLL4 and the Serrate-like ligands Jagged1 and Jagged2) and four Notch receptors (i.e., Notch1-4). Notch receptors are type-I transmembrane proteins with (i) an extracellular domain (NECD); (ii) a transmembrane domain; and (iii) an intracellular domain (NICD) that contains a RAM domain, six ankyrin repeats, and a transactivation domain $[5,6]$. The activation of Notch depends on cell-to-cell contact in which Notch ligands present on a cell bind to Notch receptors present on a neighboring cell (i.e., the signal-receiving cell) $[5,7]$ and trigger metalloproteases in the a disintegrin and metalloprotease (ADAM) family, resulting in the cleavage of the extracellular domain [8]. Subsequently, activated $\gamma$-secretase cleaves within the transmembrane domain of Notch receptors, resulting in the release of the NICD [6,9]. The NICD translocates to the nucleus and binds via its RAM and ankyrin domains to the DNA-binding transcription factor CSL (which consists of CBP or RBP-JK in vertebrates, Su (H) in Drosophila, and Lag-1 in Caenorhabditis elegans), Mastermind like-1 (MAML-1) and p300/CBP. Once formed, this complex displaces the co-repressors bound to the transcription factors, recruits transcriptional co-activators and induces the expression of target genes, such as hairyenhancer-of-split (Hes-1) and Hes-related protein gene families [5, 6, 10, 11], that subsequently execute pro-survival functions.

\section{Notch signaling and intestinal homeostasis}

Notch signaling is required for the normal maintenance and homeostasis of the intestinal epithelium $[5,12,13]$. In particular, this pathway plays an important role in controlling the cellular fate of intestinal stem cells and the differentiation of colonic goblet cells [11, 14, 15]. The expression of components of this signaling pathway has been demonstrated in both the developing and adult intestine [16,17]. Various studies demonstrated that the intestinal epithelium is enriched in the expression of Notch1, Notch2, DLL1, DLL4 and Jagged1 within the crypts. The secretory lineage of crypt cells, including the crypt base goblet cells in the colon, exhibit high levels of expression of DLL1 and DLL4 [18-20]. In the human colon, the Notch-1, -2 and -3 are highly expressed at the basal crypt while CSL and Jagged1 are highly expressed at the top of the crypts [21]. Notch signaling is crucial for the proliferation of crypt progenitors and for the differentiation of colonic epithelial cells [6]. 
Published studies demonstrate that deleting CSL/RBP-JK in combination with the deletion of Notch 1 and Notch 2 or treatment with a $\gamma$-secretase inhibitor skewed colon-based columnar stem cells to differentiate into intestinal secretory cells, primarily goblet cells [15, 22]. Conversely, in transgenic mice, ectopic expression of the NICD throughout the intestinal epithelium caused a marked decrease in secretory cell production, indicating that Notch activation leads to the amplification of the intestinal progenitor pool and the inhibition of cell differentiation $[11,23]$. In addition, Notch signaling is necessary for and works synergistically with Wnt signaling to promote the maintenance of the gut [24]. Notch and Wnt signaling act synergistically to inhibit the terminal differentiation of intestinal epithelial cells by downregulating the basic helix-loop-helix transcription factor ATOH1 (Atonal homolog 1), which is also termed MATH1 or HATH1 [25]. ATOH1 is a transcriptional activator that is repressed by HES-1; this protein is one of the most abundant targets of Notch and plays an opposing role to that of Notch/Hes by promoting secretory lineage differentiation [26-28].

\section{The importance of Notch signaling in cancer}

Notch signaling is critical for maintaining the balance between cell proliferation, differentiation, and apoptosis and is also involved in angiogenesis and the migration of cancer cells [6, 29]. Hence, deregulation of these processes that are regulated by Notch signaling may lead to the initiation and/or progression of CRC [30]. Notch receptors and their ligands are aberrantly activated in many human cancers, such as T-ALL [31, 32], pancreatic cancer [33, 34], breast cancer [35-37], prostate cancer [38-40], liver cancer [41], cervical cancer [42, 43], Kaposi's sarcoma [44], lung cancer [45], ovarian cancer [46], lymphoma [47], renal cancer [48] and CRC [49, 50]. Overexpression of Notch elements, such as receptors, ligands and downstream target genes, is correlated with increased progression, metastatic potential, and recurrence and poor prognosis and clinical outcome in various cancers [38]. For example, overexpression of Notch1 is associated with decreased time to recurrence in breast cancer [51]; similarly, high expression of Jagged-1 is correlated with the recurrence of prostate cancer [38]. Moreover, inhibiting Notch signaling with $\gamma$ secretase (GSI) in rodents caused a noticeable overproduction of goblet and enteroendocrine cells [52-54].

It was previously demonstrated that Notch is activated in primary CRC rather than metastatic colon cancer, implying that the activation of Notch may be an early step of CRC development [55]. In contrast, a more recent study reported high expression of Notch-1 and its target gene Hes-1 during both colon cancer progression [9] and metastasis [4]. No clear mechanism for the constitutive activation of Notch has been reported, and the implications of this activation for the initiation and progression of CRC remain unknown; however, mutations in the Notch receptor may play a significant role. In addition, the activity of Notch1 is also increased as a result of $\beta$-catenin-mediated upregulation of the Notch ligand Jagged-1 [56].

Notch ligand Jagged1 is highly confined to enteroendocrine cells and is undetectable in the mucosa of the small or large intestine; however, higher expression of this ligand is observed in human colon tumors (12-20). A recent study reported that downregulation of Jagged1

Curr Colorectal Cancer Rep. Author manuscript; available in PMC 2015 December 01. 
decreases cell viability and causes cell cycle arrest by downregulating the expression of Cyclin D1, Cyclin E and c-Myc in CRC [50]. These in vitro studies also demonstrated a reduction of the migratory and invasive behavior of CRC cells. Further, knocking down Jagged-1 inhibited the growth of xenograft tumors compared to controls, supporting the therapeutic role of Notch in CRC models [50]. Activation of Notch signaling has been reported to be indispensable for the development of adenomas in $\mathrm{APC}^{\mathrm{Min} /+}$ mice and for the self-renewal of tumor-initiating cells [1].

\section{Targeting Notch signaling in CRC}

Various approaches are being used to inhibit Notch signaling and are under investigation in many cancer types; this topic is discussed in detail by Espinoza and Miele [57]. These approaches include neutralizing Notch antibody, in which blocking monoclonal antibodies $(\mathrm{mAb})$ are directed against Notch receptors (i.e., Notch-1, -2, -3, -4). In addition, blocking antibodies against Notch ligands are under development. A novel mAb against the extracellular domain of nicastrin has also been generated [58]. This mAb recognizes fully mature nicastrin in the active $\gamma$-secretase complex and inhibits its activity. Another attractive therapeutic candidate is decoy, which is the soluble form of the extracellular domains of Notch receptors [59]. These decoys compete with their cell surface-bound endogenous counterparts and abolish Notch signaling, as they lack the transmembrane region that is necessary for receptor activation. In another approach, various clinical trials have focused on blocking the cleavage process of Notch receptors with $\gamma$-secretase inhibitors (GSIs) [60, 61]. $\gamma$-secretase is a promising target for Notch inhibition and exhibits cytostatic or cytotoxic activities in various cancer cells [57]. Silencing Notch1 with GSIs sensitizes colon cancer cells to chemotherapy $[9,61]$. Although GSIs appear to be attractive tools for inhibiting Notch signaling, there are some drawbacks associated with these inhibitors, as they exhibit some off-target and adverse effects, leading to gastrointestinal toxicity and liver injury [50, 62]. Another study published by Timme et al. demonstrated the nonspecific effects of a GSI [63]. GSIs are known to induce apoptosis while enhancing the response to chemotherapy in various cancers. However, in this study, the authors found that treating colon cancer cells with a potent inhibitor of $\gamma$-secretase reduced oxaliplatin-induced apoptosis by increasing the expression of anti-apoptotic proteins (i.e., Mcl-1 and/or Bcl-xL). In addition, GSI treatment alone exerted no apoptosis or growth inhibitory effect. This study is surprising and demonstrates that caution is warranted when treating colon cancer with GSIs in combination with chemotherapeutic agents.

Although many approaches are available to inhibit Notch signaling, these approaches are either in their infancy or are under investigation due to toxicity issues. Thus, it is very important to identify small molecules or establish regimens that target Notch signaling in CRC with minimal or no side effects. One such approach is the use of dietary chemopreventive agents, which might be non-toxic to normal cells/organs while selectively inhibiting tumorigenesis [64-68]. Natural products are of paramount importance for the identification of novel anticancer agents. One such plant-derived natural product is WA. This product has gained considerable scientific attention. The next section of this article will focus on WA and its anticancer properties and effects. 


\section{Withaferin A targets Notch signaling}

Withaferin A [(4 $\beta, 5 \beta, 6 \beta, 22 \mathrm{R})$-4,27-dihydroxy-5,6:22,26-diepoxyergosta-2,24-diene-1,26dione)] is a bioactive compound derived from the medicinal plant Withania somnifera Dunal. This plant is commonly known as Ashwagandha, Indian ginseng or Indian winter cherry. Withania somnifera is a small subtropical shrub, and products from this plant have been used safely for centuries in the Indian Ayurvedic system of medicine to treat various ailments and to increase longevity and vitality [68-73]. The root and leaf extracts of this plant protect against chemical-induced carcinogenesis in experimental rodents, as reviewed previously [74]. WA is a steroidal lactone and is derived from the leaves and roots of this plant [75]. The ethnobotanical history of WA-containing herbal preparations reveals the numerous effects of this compound, including anticancer and anti-inflammatory effects [76], a preventive role in neurologic disorders [69] and growth inhibitory properties in various cancer cell lines, including human colon cancer cells [77, 78]. This natural compound WA exhibits diverse pharmacologic activities (Figure 1), including anti-inflammatory, cardioactive, central nervous system, immunomodulatory and antiangiogenic effects [7981]. WA also exerts analgesic and antipyretic effects in mice [82]. WA targets vimentin in soft tissue sarcoma, suggesting a role of this compound in the modulation of epithelial to mesenchymal transitions [83].

In recent years, our laboratory explored the anticancer effects of WA on various cancer types and demonstrated the incredible potential of this natural compound as an anticancer agent [84-86]. Our results revealed an inhibition of Notch1 signaling in WA-treated CRC at both the RNA and protein levels. Notch1 inhibition also affected downstream targets, such as Hes-1 and Hey-1. Interestingly, no inhibitory effects on $\gamma$-secretase complex subunits were observed. This WA-mediated inhibition of Notch1 also affected other pro-survival signaling pathways, which are reported to be involved in crosstalk with Notch signaling. WA inhibited the AKT/NF- $\mathrm{kB} / \mathrm{Bcl}-2$ axis, thereby inhibiting cellular proliferation and inducing apoptosis in these colon cancer cells. In addition, we also observed a concomitant downregulation of mTOR signaling in WA-treated CRC cells.

The overexpression of Notch1 in colon cancer cells increased the expression of the downstream targets Hes-1 and Hey-1. In addition, this overexpression resulted in increased expression of pAKT and the mTOR signaling components pp70S6K and p-4E-BP1. In contrast, knockdown of Notch1 had the opposite effect, confirming Notch-mediated modulation of Akt and mTOR signaling. These results suggest that Notch is upstream of Akt and mTOR signaling. In addition, Notch signaling interacts with the ERK and JNK pathways. Notch negatively regulates c-Jun and JNK in various cancer types. In this study, WA treatment also upregulated phosphorylated c-Jun expression and JNK expression, thereby inducing apoptosis in colon cancer cells [84]. Similar findings were observed in breast cancer, where WA resulted in the inhibition of Notch1 [87]. In ovarian cancer [88] cells, WA downregulated expression of Notch1 and Notch3 was observed. WA treatment of CaOV3 and SKOV3 ovarian cancer cells inhibited the growth and colony formation efficiency of these cells [88]. WA also induced cell cycle arrest and apoptosis in these cancer cells. At the molecular level, these changes were accompanied by the downregulation of the Notch1, Notch3, cdc25C, total Akt, phosphorylated Akt and bcl-2 proteins. This study 
suggests that WA is a potential therapeutic agent for ovarian cancer. Consistent with these studies that investigated WA and Notch signaling, our unpublished data demonstrate that WA inhibits tumor growth in xenograft models of colon cancer.

\section{Conclusions and future directions}

Notch signaling is an important therapeutic target, as it plays a major role in the colonic crypt compartment by maintaining colon homeostasis via the regulation of colon stem cell behavior and differentiation. However, aberrant activation of Notch initiates and promotes colon carcinogenesis, hence selectively targeting Notch signaling in colon cancer cells could be an ideal strategy for prevention and treatment of colon cancer. WA appears to have tremendous anticancer potential as a result of targeting multiple molecules in a variety of human cancers, including $\mathrm{CRC}$, as evidenced by various preclinical in vitro and in vivo studies. Only our group has demonstrated the Notch inhibitory role of WA in CRC. It will be interesting to study the effect of this molecule in colon cancer stem cells that reside in the colon crypts and play an important role in this disease. In addition, the effect of this natural compound in combination with current chemotherapeutic drugs must be studied. We are currently generating potent WA analogs, which could be even more effective in inhibiting Notch signaling than WA. Appropriately powered studies are needed to take this molecule, which has been known to have therapeutic value for decades, from bench to bedside in the clinical setting.

\section{Acknowledgments}

This study was supported by NIH Grant 1R01CA185972-01.

\section{References}

Papers of particular interest, published recently, have been highlighted as:

- Of importance

•- Of major importance

1. Miyamoto S, Rosenberg DW. Role of Notch signaling in colon homeostasis and carcinogenesis. Cancer Sci. 2011; 102(11):1938-42. [PubMed: 21801279]

2. Jemal A, et al. Global cancer statistics. CA Cancer J Clin. 2011; 61(2):69-90. [PubMed: 21296855]

3. Siegel R, Desantis C, Jemal A. Colorectal cancer statistics, 2014. CA Cancer J Clin. 2014; 64(2): 104-17. [PubMed: 24639052]

4. Wu WK, et al. Dysregulation and crosstalk of cellular signaling pathways in colon carcinogenesis. Crit Rev Oncol Hematol. 2013; 86(3):251-77. [PubMed: 23287077]

5•. Noah TK, Shroyer NF. Notch in the intestine: regulation of homeostasis and pathogenesis. Annu Rev Physiol. 2013; 75:263-88. This article describe the role of Notch in intestinal homeostasis and colorectal cancer. [PubMed: 23190077]

6. Qiao L, Wong BC. Role of Notch signaling in colorectal cancer. Carcinogenesis. 2009; 30(12): 1979-86. [PubMed: 19793799]

7. Barolo S, Posakony JW. Three habits of highly effective signaling pathways: principles of transcriptional control by developmental cell signaling. Genes Dev. 2002; 16(10):1167-81. [PubMed: 12023297]

8. van Tetering G, et al. Metalloprotease ADAM10 is required for Notch1 site 2 cleavage. J Biol Chem. 2009; 284(45):31018-27. [PubMed: 19726682] 
9. Meng RD, et al. gamma-Secretase inhibitors abrogate oxaliplatin-induced activation of the Notch-1 signaling pathway in colon cancer cells resulting in enhanced chemosensitivity. Cancer Res. 2009; 69(2):573-82. [PubMed: 19147571]

10. Blaumueller CM, et al. Intracellular cleavage of Notch leads to a heterodimeric receptor on the plasma membrane. Cell. 1997; 90(2):281-91. [PubMed: 9244302]

11. Fre $\mathrm{S}$, et al. Notch signals control the fate of immature progenitor cells in the intestine. Nature. 2005; 435(7044):964-8. [PubMed: 15959516]

12. Pellegrinet L, et al. Dll1- and dll4-mediated notch signaling are required for homeostasis of intestinal stem cells. Gastroenterology. 2011; 140(4):1230-1240. e1-7. [PubMed: 21238454]

13. Radtke F, Clevers H, Riccio O. From gut homeostasis to cancer. Curr Mol Med. 2006; 6(3):27589. [PubMed: 16712475]

14. Zheng H, et al. KLF4 gene expression is inhibited by the notch signaling pathway that controls goblet cell differentiation in mouse gastrointestinal tract. Am J Physiol Gastrointest Liver Physiol. 2009; 296(3):G490-8. [PubMed: 19109406]

15. Riccio O, et al. Loss of intestinal crypt progenitor cells owing to inactivation of both Notch1 and Notch2 is accompanied by derepression of CDK inhibitors p27Kip1 and p57Kip2. EMBO Rep. 2008; 9(4):377-83. [PubMed: 18274550]

16. Schroder N, Gossler A. Expression of Notch pathway components in fetal and adult mouse small intestine. Gene Expr Patterns. 2002; 2(3-4):247-50. [PubMed: 12617809]

17. Sander GR, Powell BC. Expression of notch receptors and ligands in the adult gut. J Histochem Cytochem. 2004; 52(4):509-16. [PubMed: 15034002]

18. VanDussen KL, et al. Notch signaling modulates proliferation and differentiation of intestinal crypt base columnar stem cells. Development. 2012; 139(3):488-97. [PubMed: 22190634]

19. Vooijs M, et al. Mapping the consequence of Notch1 proteolysis in vivo with NIP-CRE. Development. 2007; 134(3):535-44. [PubMed: 17215306]

20. Fre $S$, et al. Notch lineages and activity in intestinal stem cells determined by a new set of knock-in mice. PLoS One. 2011; 6(10):e25785. [PubMed: 21991352]

21. Kosinski C, et al. Gene expression patterns of human colon tops and basal crypts and BMP antagonists as intestinal stem cell niche factors. Proc Natl Acad Sci U S A. 2007; 104(39):1541823. [PubMed: 17881565]

22. van Es JH, et al. Notch/gamma-secretase inhibition turns proliferative cells in intestinal crypts and adenomas into goblet cells. Nature. 2005; 435(7044):959-63. [PubMed: 15959515]

23. Stanger BZ, et al. Direct regulation of intestinal fate by Notch. Proc Natl Acad Sci U S A. 2005; 102(35):12443-8. [PubMed: 16107537]

24. Fre S, et al. Notch and Wnt signals cooperatively control cell proliferation and tumorigenesis in the intestine. Proc Natl Acad Sci U S A. 2009; 106(15):6309-14. [PubMed: 19251639]

25. Katoh M, Katoh M. Notch signaling in gastrointestinal tract (review). Int J Oncol. 2007; 30(1): 247-51. [PubMed: 17143535]

26. Shroyer NF, et al. Intestine-specific ablation of mouse atonal homolog 1 (Math1) reveals a role in cellular homeostasis. Gastroenterology. 2007; 132(7):2478-88. [PubMed: 17570220]

27. Yang Q, et al. Requirement of Math1 for secretory cell lineage commitment in the mouse intestine. Science. 2001; 294(5549):2155-8. [PubMed: 11739954]

28. Zheng X, et al. Suppression of hath1 gene expression directly regulated by hes1 via notch signaling is associated with goblet cell depletion in ulcerative colitis. Inflamm Bowel Dis. 2011; 17(11): 2251-60. [PubMed: 21987298]

29. Schroeter EH, Kisslinger JA, Kopan R. Notch-1 signalling requires ligand-induced proteolytic release of intracellular domain. Nature. 1998; 393(6683):382-6. [PubMed: 9620803]

30. Yin L, Velazquez OC, Liu ZJ. Notch signaling: emerging molecular targets for cancer therapy. Biochem Pharmacol. 2010; 80(5):690-701. [PubMed: 20361945]

31. Ellisen LW, et al. TAN-1, the human homolog of the Drosophila notch gene, is broken by chromosomal translocations in T lymphoblastic neoplasms. Cell. 1991; 66(4):649-61. [PubMed: 1831692] 
32. Pear WS, et al. Exclusive development of $\mathrm{T}$ cell neoplasms in mice transplanted with bone marrow expressing activated Notch alleles. J Exp Med. 1996; 183(5):2283-91. [PubMed: 8642337]

33. Kimura K, et al. Activation of Notch signaling in tumorigenesis of experimental pancreatic cancer induced by dimethylbenzanthracene in mice. Cancer Sci. 2007; 98(2):155-62. [PubMed: 17297654]

34. Wang Z, et al. Down-regulation of notch-1 inhibits invasion by inactivation of nuclear factorkappaB, vascular endothelial growth factor, and matrix metalloproteinase-9 in pancreatic cancer cells. Cancer Res. 2006; 66(5):2778-84. [PubMed: 16510599]

35. Mao J, et al. ShRNA targeting Notch1 sensitizes breast cancer stem cell to paclitaxel. Int J Biochem Cell Biol. 2013; 45(6):1064-73. [PubMed: 23500524]

36. Reedijk M, et al. High-level coexpression of JAG1 and NOTCH1 is observed in human breast cancer and is associated with poor overall survival. Cancer Res. 2005; 65(18):8530-7. [PubMed: 16166334]

37. Suman S, Das TP, Damodaran C. Silencing NOTCH signaling causes growth arrest in both breast cancer stem cells and breast cancer cells. Br J Cancer. 2013; 109(10):2587-96. [PubMed: 24129237]

38. Santagata S, et al. JAGGED1 expression is associated with prostate cancer metastasis and recurrence. Cancer Res. 2004; 64(19):6854-7. [PubMed: 15466172]

39. Marignol L. Targeting notch in prostate cancer-combination is the key. Nat Rev Urol. 2014; 11(7): 419. [PubMed: 24980190]

40. Kwon OJ, et al. Increased Notch signalling inhibits anoikis and stimulates proliferation of prostate luminal epithelial cells. Nat Commun. 2014; 5:4416. [PubMed: 25048699]

41. Gao J, et al. Expression of Jagged1 and its association with hepatitis B virus X protein in hepatocellular carcinoma. Biochem Biophys Res Commun. 2007; 356(2):341-7. [PubMed: 17359939]

42. Tripathi R, et al. Clinical impact of de-regulated Notch-1 and Notch-3 in the development and progression of HPV-associated different histological subtypes of precancerous and cancerous lesions of human uterine cervix. PLoS One. 2014; 9(6):e98642. [PubMed: 24905589]

43. Ramdass B, et al. Coexpression of Notch1 and NF-kappaB signaling pathway components in human cervical cancer progression. Gynecol Oncol. 2007; 104(2):352-61. [PubMed: 17098279]

44. Curry CL, et al. Notch inhibition in Kaposi's sarcoma tumor cells leads to mitotic catastrophe through nuclear factor-kappaB signaling. Mol Cancer Ther. 2007; 6(7):1983-92. [PubMed: 17604336]

45. Konishi J, et al. Gamma-secretase inhibitor prevents Notch3 activation and reduces proliferation in human lung cancers. Cancer Res. 2007; 67(17):8051-7. [PubMed: 17804716]

46. Wang H, et al. The expression of VEGF and Dll4/Notch pathway molecules in ovarian cancer. Clin Chim Acta. 2014; 436C:243-248. [PubMed: 24949865]

47. Jundt F, et al. Activated Notch1 signaling promotes tumor cell proliferation and survival in Hodgkin and anaplastic large cell lymphoma. Blood. 2002; 99(9):3398-403. [PubMed: 11964309]

48. Sjolund J, et al. Suppression of renal cell carcinoma growth by inhibition of Notch signaling in vitro and in vivo. J Clin Invest. 2008; 118(1):217-28. [PubMed: 18079963]

49. Ozawa T, et al. Nuclear Notch3 Expression is Associated with Tumor Recurrence in Patients with Stage II and III Colorectal Cancer. Ann Surg Oncol. 2014; 21(8):2650-8. [PubMed: 24728738]

50. Dai Y, et al. Silencing of Jagged1 inhibits cell growth and invasion in colorectal cancer. Cell Death Dis. 2014; 5:e1170. [PubMed: 24722295]

51. Farnie G, et al. Novel cell culture technique for primary ductal carcinoma in situ: role of Notch and epidermal growth factor receptor signaling pathways. J Natl Cancer Inst. 2007; 99(8):616-27. [PubMed: 17440163]

52. Wong GT, et al. Chronic treatment with the gamma-secretase inhibitor LY-411,575 inhibits betaamyloid peptide production and alters lymphopoiesis and intestinal cell differentiation. J Biol Chem. 2004; 279(13):12876-82. [PubMed: 14709552]

53. Milano J, et al. Modulation of notch processing by gamma-secretase inhibitors causes intestinal goblet cell metaplasia and induction of genes known to specify gut secretory lineage differentiation. Toxicol Sci. 2004; 82(1):341-58. [PubMed: 15319485] 
54. Searfoss GH, et al. Adipsin, a biomarker of gastrointestinal toxicity mediated by a functional gamma-secretase inhibitor. J Biol Chem. 2003; 278(46):46107-16. [PubMed: 12949072]

55. Veenendaal LM, et al. Differential Notch and TGFbeta signaling in primary colorectal tumors and their corresponding metastases. Cell Oncol. 2008; 30(1):1-11. [PubMed: 18219106]

56. Rodilla V, et al. Jagged1 is the pathological link between Wnt and Notch pathways in colorectal cancer. Proc Natl Acad Sci U S A. 2009; 106(15):6315-20. [PubMed: 19325125]

57•. Espinoza I, Miele L. Notch inhibitors for cancer treatment. Pharmacol Ther. 2013; 139(2):95-110. This review article describes the role of Notch signaling and apporoaches inhibit this signaling in various cancers. [PubMed: 23458608]

58. Hayashi I, et al. Neutralization of the gamma-secretase activity by monoclonal antibody against extracellular domain of nicastrin. Oncogene. 2012; 31(6):787-98. [PubMed: 21725355]

59. Funahashi $Y$, et al. A notch1 ectodomain construct inhibits endothelial notch signaling, tumor growth, and angiogenesis. Cancer Res. 2008; 68(12):4727-35. [PubMed: 18559519]

60. Singh A, et al. GSI promotes vincristine-induced apoptosis by enhancing multipolar spindle formation. Cell Cycle. 2014; 13(1):157-66. [PubMed: 24200971]

61. Akiyoshi T, et al. Gamma-secretase inhibitors enhance taxane-induced mitotic arrest and apoptosis in colon cancer cells. Gastroenterology. 2008; 134(1):131-44. [PubMed: 18166351]

62••. Takebe N, Nguyen D, Yang SX. Targeting notch signaling pathway in cancer: clinical development advances and challenges. Pharmacol Ther. 2014; 141(2):140-9. This recent review article describes the role of Notch signaling in cancer as well as the agents in use to target Notch signaling in different cancer types. [PubMed: 24076266]

63••. Timme CR, Gruidl M, Yeatman TJ. Gamma-secretase inhibition attenuates oxaliplatin-induced apoptosis through increased Mcl-1 and/or Bcl-xL in human colon cancer cells. Apoptosis. 2013; 18(10):1163-74. It is a interesting study where authors have shown gamma-secretase inhibition resulted in decrease in oxaliplatin-induced apoptosis. This study warrants caution while treating colon cancer with the combination of GSIs and chemotherapy. [PubMed: 23887890]

64. Herszenyi L, et al. Chemoprevention of colorectal cancer: feasibility in everyday practice? Eur J Cancer Prev. 2008; 17(6):502-14. [PubMed: 18941372]

65. Cooper K, et al. Chemoprevention of colorectal cancer: systematic review and economic evaluation. Health Technol Assess. 2010; 14(32):1-206.

66. Gwyn K, Sinicrope FA. Chemoprevention of colorectal cancer. Am J Gastroenterol. 2002; 97(1): 13-21. [PubMed: 11808936]

67. Surh YJ. Cancer chemoprevention with dietary phytochemicals. Nat Rev Cancer. 2003; 3(10):76880. [PubMed: 14570043]

68. Vadodkar AS, et al. Chemoprevention of breast cancer by dietary compounds. Anticancer Agents Med Chem. 2012; 12(10):1185-202. [PubMed: 22583403]

69. Mishra LC, Singh BB, Dagenais S. Scientific basis for the therapeutic use of Withania somnifera (ashwagandha): a review. Altern Med Rev. 2000; 5(4):334-46. [PubMed: 10956379]

70. Malik F, et al. A standardized root extract of Withania somnifera and its major constituent withanolide-A elicit humoral and cell-mediated immune responses by up regulation of Th1dominant polarization in BALB/c mice. Life Sci. 2007; 80(16):1525-38. [PubMed: 17336338]

71. Malik F, et al. Reactive oxygen species generation and mitochondrial dysfunction in the apoptotic cell death of human myeloid leukemia HL-60 cells by a dietary compound withaferin A with concomitant protection by $\mathrm{N}$-acetyl cysteine. Apoptosis. 2007; 12(11):2115-33. [PubMed: 17874299]

72. Malik F, et al. Immune modulation and apoptosis induction: Two sides of antitumoural activity of a standardised herbal formulation of Withania somnifera. Eur J Cancer. 2009; 45(8):1494-509. [PubMed: 19269163]

73. Winters M. Ancient medicine, modern use: Withania somnifera and its potential role in integrative oncology. Altern Med Rev. 2006; 11(4):269-77. [PubMed: 17176166]

74••. Vyas AR, Singh SV. Molecular targets and mechanisms of cancer prevention and treatment by withaferin a, a naturally occurring steroidal lactone. AAPS J. 2014; 16(1):1-10. The authors have discussed the in vivo potential and molecular targets of WA contributing to its anticancer effects. [PubMed: 24046237] 
75. Mirjalili MH, et al. Steroidal lactones from Withania somnifera, an ancient plant for novel medicine. Molecules. 2009; 14(7):2373-93. [PubMed: 19633611]

76. Bhattacharya SK, Satyan KS, Ghosal S. Antioxidant activity of glycowithanolides from Withania somnifera. Indian J Exp Biol. 1997; 35(3):236-9. [PubMed: 9332168]

77. Kaileh M, et al. Withaferin a strongly elicits IkappaB kinase beta hyperphosphorylation concomitant with potent inhibition of its kinase activity. J Biol Chem. 2007; 282(7):4253-64. [PubMed: 17150968]

78. Jayaprakasam B, et al. Growth inhibition of human tumor cell lines by withanolides from Withania somnifera leaves. Life Sci. 2003; 74(1):125-32. [PubMed: 14575818]

79. Mohan R, et al. Withaferin A is a potent inhibitor of angiogenesis. Angiogenesis. 2004; 7(2):11522. [PubMed: 15516832]

80. Bhattacharya SK, et al. Effect of Withania somnifera glycowithanolides on a rat model of tardive dyskinesia. Phytomedicine. 2002; 9(2):167-70. [PubMed: 11995951]

81. Chowdhury K, Neogy RK. Mode of action of Withaferin A and Withanolide D. Biochem Pharmacol. 1975; 24(8):919-20. [PubMed: 1125091]

82. Sabina PE, Chandel S, Raool KM. Evaluation of analgesic, antipyretic and ulcerogenice effect of Withaferin A. International journal of integrative biology. 2009; 6(2):52-56.

83. Lahat $\mathrm{G}$, et al. Vimentin is a novel anti-cancer therapeutic target; insights from in vitro and in vivo mice xenograft studies. PLoS One. 2010; 5(4):e10105. [PubMed: 20419128]

84. Koduru S, et al. Notch-1 inhibition by Withaferin-A: a therapeutic target against colon carcinogenesis. Mol Cancer Ther. 2010; 9(1):202-10. [PubMed: 20053782]

85. Srinivasan S, et al. Par-4-dependent apoptosis by the dietary compound withaferin A in prostate cancer cells. Cancer Res. 2007; 67(1):246-53. [PubMed: 17185378]

86. Roy RV, et al. Withaferin A, a steroidal lactone from Withania somnifera, induces mitotic catastrophe and growth arrest in prostate cancer cells. J Nat Prod. 2013; 76(10):1909-15. [PubMed: 24079846]

87. Lee J, Sehrawat A, Singh SV. Withaferin A causes activation of Notch2 and Notch4 in human breast cancer cells. Breast Cancer Res Treat. 2012; 136(1):45-56. Authors have shown the effect of WA in breast cancer cells. They showed activation of Notch 2,4 and inactivation of Notch1 by WA. Noch2 and Notch4 knockdown augmented WA mediated inhibition of cell migration. [PubMed: 22965833]

88. Zhang X, et al. Inhibition of cell growth and induction of apoptosis in ovarian carcinoma cell lines CaOV3 and SKOV3 by natural withanolide Withaferin A. Gynecol Oncol. 2012; 124(3):606-12. Authors have shown WA mediated downregulation of Notch1 and 3 in ovarian cancer cells. This downregulation correlated with induction of apoptosis and cell cycle arrest. [PubMed: 22188785] 


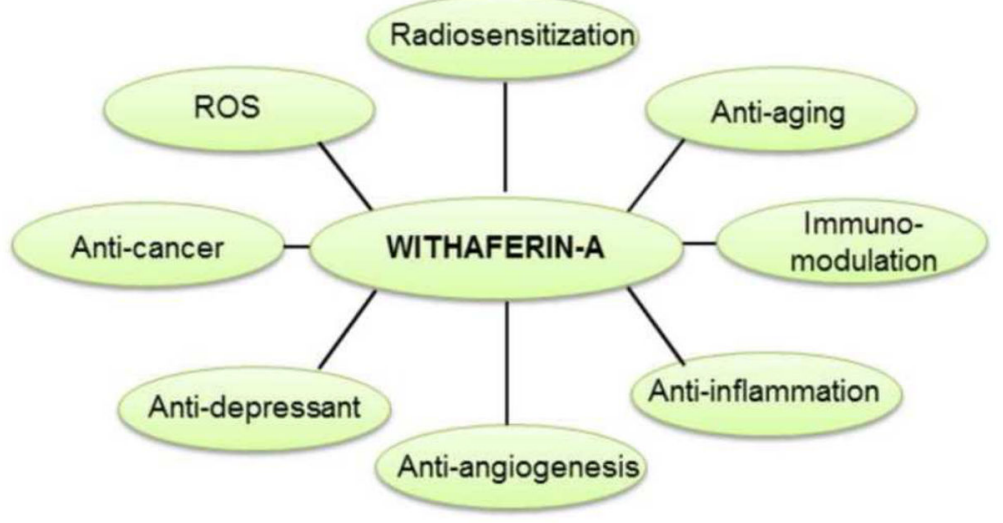

Figure 1.

Medicinal properties of Withaferin-A 